\title{
Arctic Stratosphere Dynamical Response to Global Warming
}

\author{
ALEXEY YU. KARPECHKO \\ Arctic Research, Finnish Meteorological Institute, Helsinki, Finland \\ ELISA MANZINI \\ Max Planck Institute for Meteorology, Hamburg, Germany
}

(Manuscript received 30 October 2016, in final form 22 May 2017)

\begin{abstract}
The role of stationary planetary waves in the dynamical response of the Arctic winter stratosphere circulation to global warming is investigated here by analyzing simulations performed with atmosphere-only models from phase 5 of the Coupled Model Intercomparison Project (CMIP5) driven by prescribed sea surface temperatures (SSTs). Climate models often simulate dynamical warming of the Arctic stratosphere as a response to global warming in association with a strengthening of the deep branch of the Brewer-Dobson circulation; however, until now, no satisfactory mechanism for such a response has been suggested. This study focuses on December-February (DJF) because this is the period when the troposphere and stratosphere are strongly coupled. When forced by increased SSTs, all the models analyzed here simulate Arctic stratosphere dynamical warming, mostly due to increased upward propagation of quasi-stationary wavenumber 1 , as diagnosed by the meridional eddy heat flux. Further, it is shown that the stratospheric warming and increased wave flux to the stratosphere are related to the strengthening of the zonal winds in subtropics and midlatitudes near the tropopause. Evidence presented in this paper corroborate climate model simulations of future stratospheric changes and suggest a dynamical warming of the Arctic polar vortex as the most likely response to global warming.
\end{abstract}

\section{Introduction}

The stratospheric meridional circulation, also known as the Brewer-Dobson (BD) circulation, is characterized by ascent in the tropics and descent in the extratropics [see the review by Butchart (2014)]. It transfers ozone from its source region in the tropics to the extratropics, which has a profound impact on surface UV radiations (e.g., Fioletov et al. 1997) and air quality (e.g., Neu et al. 2014). The BD circulation is driven by the dissipation of planetary and gravity waves (Holton et al. 1995) and is often separated into shallow (below $\sim 70 \mathrm{hPa}$ ) and deep (above $\sim 70 \mathrm{hPa}$ ) branches (Plumb 2002; Birner and Bönisch 2011). Descending motion associated with the deep branch of the BD circulation is

Supplemental information related to this paper is available at the Journals Online website: http://dx.doi.org/10.1175/ JCLI-D-16-0781.s1.

Corresponding author: A. Yu. Karpechko, alexey.karpechko@ fmi.fi responsible, in particular, for dynamical warming of the stratospheric polar vortex. Its intensity strongly (anti) correlates with the strength of the polar vortex, which affects tropospheric circulation below and influences surface climate [see the review by Kidston et al. (2015)].

Climate models typically predict strengthening of the overall BD circulation (Butchart 2014; Manzini et al. 2014) in response to the global warming, as diagnosed by the tropical mass flux at $70 \mathrm{hPa}$. The BD circulation strengthening leads to a decrease of tropical ozone and a superrecovery of extratropical ozone (Eyring et al. 2007), decreases of UV radiation in the extratropics, and increases in extratropical stratosphere-troposphere ozone flux (Hegglin and Shepherd 2009). If the BD circulation strengthening extends to the poles (i.e., it encompasses the deep branch of the BD circulation) an equatorward shift of the tropospheric eddy-driven jet streams during Northern Hemisphere $(\mathrm{NH})$ winter is also often simulated (Scaife et al. 2012; Karpechko and Manzini 2012), although it is not a robust response across the models. Butchart et al. (2000) reported that the forced response of the Arctic stratosphere to global 
warming is small compared to internal variability. Sigmond and Scinocca (2010) found that the response of the NH polar vortex depends on the basic state, which influences the propagation of the wave activity within the stratosphere. More recently, Manzini et al. (2014) analyzed models from phase 5 of the Coupled Model Intercomparison Project (CMIP5) and reported that, while all models (including models with top level below the stratopause) in the RCP8.5 scenario simulate increased upwelling in the tropics indicating strengthening of the overall BD circulation, only about $70 \%$ of the models simulate weakening of the $\mathrm{NH}$ polar night jet. The reasons for the disagreement among the models remain unclear. Moreover, in observations strengthening of the BD circulation has only been detected in the Southern Hemisphere (Fu et al. 2015) but not in the Northern Hemisphere (Engel et al. 2009; Fu et al. 2015).

Several mechanisms have been proposed to explain strengthening of the BD circulation. Eichelberger and Hartmann (2005) proposed that the strengthening is caused by increased generation and upward propagation of wave activity associated with transient wavenumber 2. However, the relevance of this mechanism is unclear, given that the increased generation of wavenumber 2 activity in their simplified model experiment was induced by unrealistically large prescribed changes in baroclinicity. Several other studies, including Olsen et al. (2007), argued that the strengthening is related to changes in refractive properties of the background flow, which causes waves to propagate more poleward, dissipate, and so strengthen the BD circulation toward the polar stratosphere. However, this mechanism is not robust across the models, likely because of strong dependence of the refraction changes on biases in the background flow (Sigmond and Scinocca 2010). More recently, Oberländer-Hayn et al. (2016) proposed that future changes of the BD circulation can be understood as an upward shift of the circulation rather than as a strengthening; however, their mechanism does not explain increases in planetary wave activity seen in several experiments (Sigmond et al. 2004; McLandress and Shepherd 2009). Most of the other studies focused on changes in the subtropics (e.g., Garcia and Randel 2008; Calvo and Garcia 2009) where a robust mechanism explaining the strengthening of the shallow branch of the BD circulation has been proposed (Shepherd and McLandress 2011). However, the mechanisms of the response of the deep branch of the circulation remain unclear (McLandress and Shepherd 2009).

The purpose of this work is to ask if changes in stationary planetary waves can explain the Arctic winter stratosphere circulation response to global warming in the multimodel ensembles of CMIP5 models with prescribed sea surface temperatures (SSTs). (See descriptions of the experiments in section 2.) We focus on December-February (DJF) because this is the period when the troposphere and stratosphere are strongly coupled. Manzini et al. (2014) found that climate sensitivity is one of the factors influencing the tropospheric dynamical response and hence possibly also the Arctic stratosphere circulation response. Grise and Polvani (2014) showed that the extratropical circulation response to global warming is dominated by a component associated with SST warming, and Woollings et al. (2012) suggested that the tropospheric dynamical response is shaped by SST response patterns, which, in general, differ across the models. By analyzing multimodel simulations with prescribed SSTs we aim to avoid uncertainty due to poorly constrained climate sensitivity and SST response and instead better understand robust circulation responses in the extratropical stratosphere to aspects of climate change such as the strengthening of the subtropical jet stream. Additional motivation to use multimodel simulations with prescribed SSTs is to isolate the Arctic stratosphere circulation response to global warming, independently of the radiative response of the stratosphere, to the increase in greenhouse gases and so generalize previous single model results such as Oberländer et al. (2013). The data are described in section 2 , the results are reported in section 3 , and conclusions are drawn in section 4.

\section{Data and methods}

CMIP5 has four experiments with atmosphere-only models forced by prescribed SSTs. First, the standard Atmospheric Model Intercomparison Project (AMIP) experiment has SSTs varying according to observations. The period of simulations is at least 1979-2008 or even longer for some models-see Table 1 for information about each model. The other three experiments represent different climate change scenarios. In the AMIP4K experiment, the SSTs from the AMIP experiment have been uniformly increased by $4 \mathrm{~K}$ globally. In AMIPFuture, the anomaly pattern corresponding to an SST response to a quadrupled $\mathrm{CO}_{2}$ concentration in the future has been added to the AMIP SSTs. The SST pattern in the AMIPFuture experiment is based on simulations from phase 3 of the Coupled Model Intercomparison Project (CMIP3) with coupled atmosphere-ocean models (see http://cfmip.metoffice.com/CMIP5.html for more details), but its magnitude is normalized so that the global mean warming is equal to $4 \mathrm{~K}$ (i.e., the same as in AMIP4K). The key differences between AMIP4K and AMIPFuture include smaller warming over the North Atlantic and the Southern Ocean and 
TABLE 1. Models and simulations used in the study. Also shown is the number of available simulations for each model

\begin{tabular}{|c|c|c|c|c|c|}
\hline Model name & Institute & Period & AMIP4K & AMIPFuture & $\mathrm{AMIP} 4 \times \mathrm{CO}_{2}$ \\
\hline BCC_CSM1.1 & Beijing Climate Center (China) & 1979-2008 & 1 & 1 & 1 \\
\hline CanAM4 & $\begin{array}{l}\text { Canadian Centre for Climate Modelling } \\
\text { and Analysis (Canada) }\end{array}$ & 1950-2009 & 1 & 1 & 1 \\
\hline CCSM4 & National Center for Atmospheric Research & 1979-2008 & 1 & 1 & - \\
\hline CNRM-CM5 & $\begin{array}{l}\text { Centre National de Recherches } \\
\text { Météorologiques (France) }\end{array}$ & 1979-2008 & 1 & 1 & 1 \\
\hline FGOALS-g2 & $\begin{array}{l}\text { State Key Laboratory of Numerical Modeling } \\
\text { for Atmospheric Sciences and Geophysical } \\
\text { Fluid Dynamics/Institute of Atmospheric } \\
\text { Physics (LASG/IAP), Chinese Academy of } \\
\text { Sciences, and Center of Experts for Services } \\
\text { and Solutions (CESS) (China) }\end{array}$ & 1979-2009 & 1 & - & 1 \\
\hline HadGEM2-A & Met Office Hadley Centre (United Kingdom) & 1979-2008 & 1 & 1 & 1 \\
\hline IPSL-CM5A-LR & L'Institut Pierre-Simon Laplace (France) & 1979-2009 & 2 & 2 & 2 \\
\hline IPSL-CM5B-LR & L'Institut Pierre-Simon Laplace (France) & 1979-2008 & 1 & 1 & 1 \\
\hline MIROC5 & $\begin{array}{l}\text { Japan Agency for Marine-Earth Science and } \\
\text { Technology, Atmosphere and Ocean Research } \\
\text { Institute, and National Institute for } \\
\text { Environmental Studies (Japan) }\end{array}$ & 1979-2008 & 1 & 1 & 1 \\
\hline MPI-ESM-LR & Max Planck Institute for Meteorology (Germany) & 1979-2008 & 1 & 1 & 1 \\
\hline MPI-ESM-MR & Max Planck Institute for Meteorology (Germany) & 1979-2008 & 1 & 1 & 1 \\
\hline MRI-CGCM3 & Meteorological Research Institute (Japan) & 1979-2009 & 1 & 1 & 1 \\
\hline
\end{tabular}

stronger warming over the tropical oceans and the North Pacific in AMIPFuture. In both experiments greenhouse gas (GHG) concentrations are kept the same as in AMIP. In AMIP4 $\times \mathrm{CO}_{2}$, the atmospheric $\mathrm{CO}_{2}$ concentration has been quadrupled while the SSTs have not been changed. In all experiments the sea ice concentrations are the same as in the AMIP experiment and correspond to the observed values. Overall 12 CMIP5 models have performed AMIP experiments and at least one climate change scenario experiment; 10 models have performed all four experiments. The models and period of simulations are listed in Table 1. All models, except IPSL-CM5A-LR, performed only one simulation for each of the climate change experiments. For consistency we use only the first ensemble member of IPSL-CM5A-LR everywhere except in sections $3 \mathrm{a}$ and $3 d$ where we use both ensemble members from this model to assess the role of internal variability.

\section{Results}

\section{a. Changes in zonal mean circulation}

Figure 1 shows multimodel mean anomalies with respect to the AMIP experiment in air temperature and zonal winds for the three climate change experiments. The AMIP4K and AMIPFuture experiments show dipole changes in stratospheric temperatures with cooling in the tropics and warming in the extratropics. In general the temperatures are subject to both radiative and dynamical changes; however, such a dipole structure suggests that the dynamical factors dominate (Yulaeva et al. 1994). Namely, the pattern indicates strengthening of the BD circulation with the ascending branch cooling the topics via adiabatic expansion and the descending branch warming the extratropics via adiabatic compression. Looking at wind changes, we find the weakening of the stratospheric and tropospheric subpolar winds, which has previously been shown to occur in other CMIP5 scenarios (Manzini et al. 2014) and in stratosphere-resolving chemistry-climate models (Scaife et al. 2012). The easterly wind anomaly in the troposphere has previously been suggested to result from the Arctic amplification due to the thermal wind relation (Woollings 2008; Deser et al. 2010) as well as being influenced by the stratospheric changes (Scaife et al. 2012; Manzini et al. 2014). In the experiments analyzed here the Arctic amplification is weak (less than $2 \mathrm{~K}$; Figs. 1b,d) owing to the lack of changes in the sea ice and thus is very unlikely to be the cause of the easterly anomalies. Although direct comparison with coupled simulations having Arctic amplification, such as those from Manzini et al. (2014), is problematic owing to different forcing and model selection, it is worth noting that in the coupled runs the easterly response in the troposphere peaks at $65^{\circ} \mathrm{N}$ whereas in our AMIP simulations it peaks at $75^{\circ} \mathrm{N}$. In terms of magnitude the tropospheric responses in both ensembles are comparable with maximum easterly response of about $1 \mathrm{~ms}^{-1}$. Both AMIP4K and AMIPFuture show strengthening of the 
a) amip4K ua $(\mathrm{m} / \mathrm{s})$

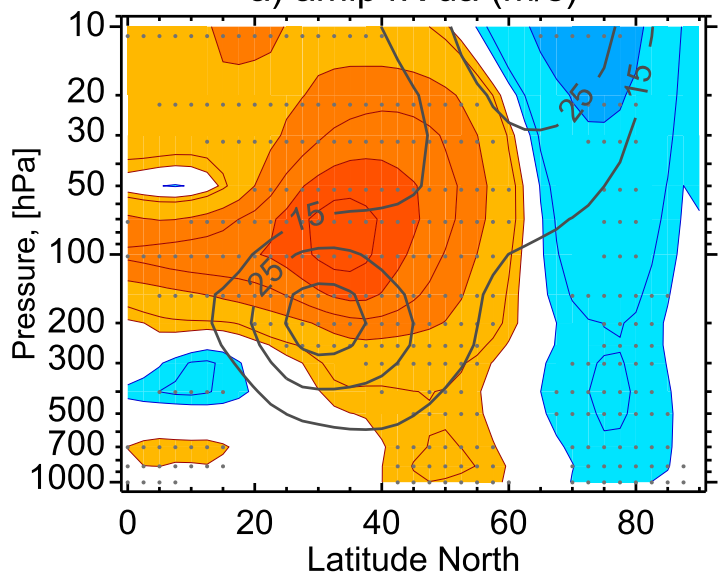

c) amipFuture ua $(\mathrm{m} / \mathrm{s})$

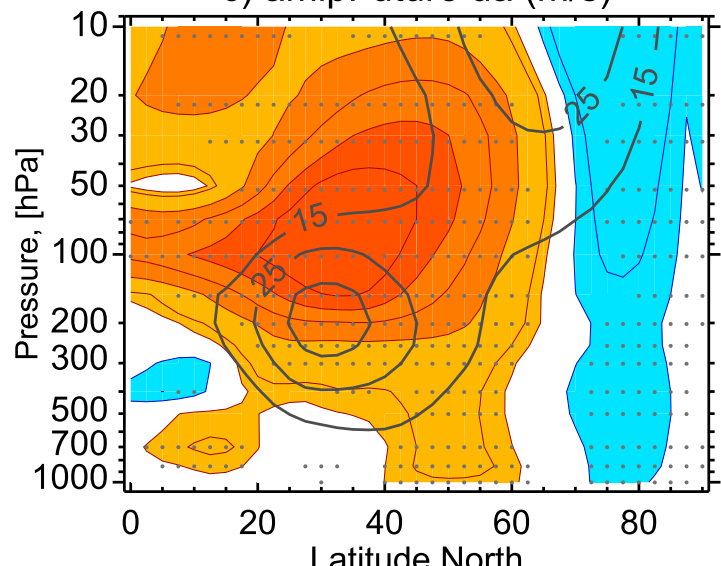

e) amip4xCO2 ua $(\mathrm{m} / \mathrm{s})$
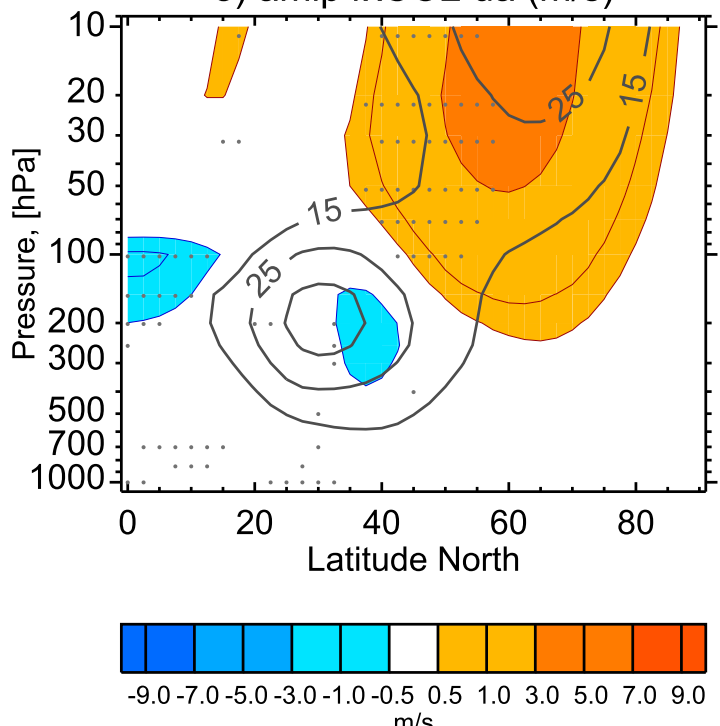

b) amip4K ta $(\mathrm{K})$

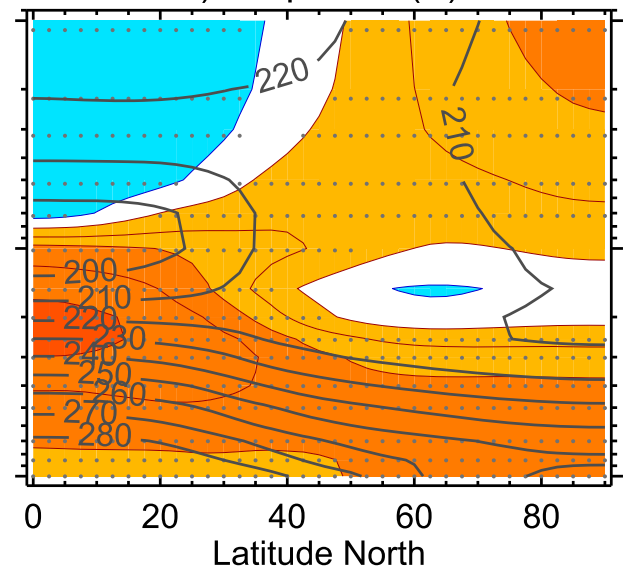

d) amipFuture ta (K)

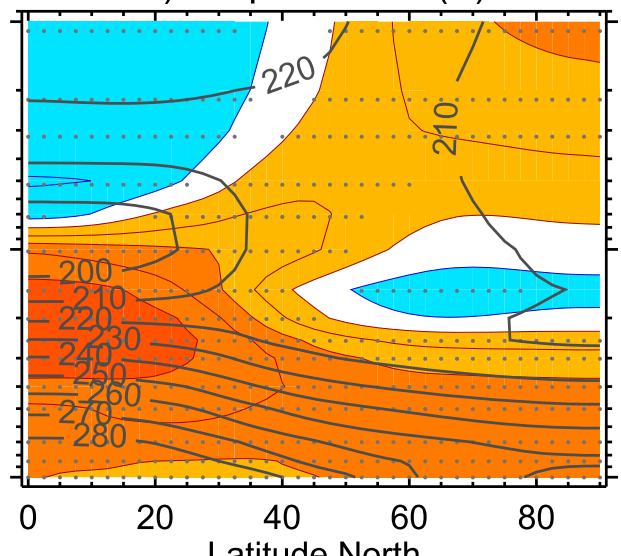

f) amip4xCO2 ta (K)
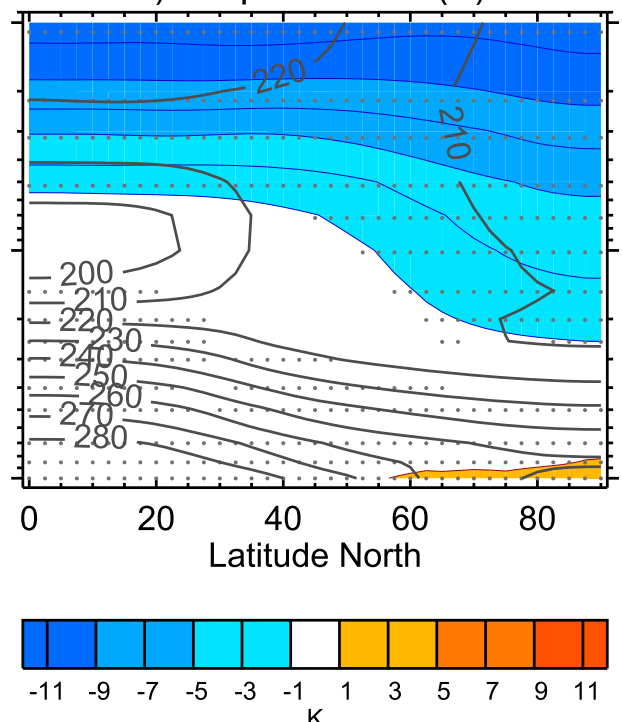

FIG. 1. Multimodel mean changes in DJF zonal mean zonal winds and temperatures in the AMIP4K, AMIPFuture, and AMIP $4 \times \mathrm{CO}_{2}$ experiments. Gray contours indicate the multimodel mean climatologies based on the AMIP experiment. Gray dots indicate grid points where more than $90 \%$ of the models (i.e., at least 11 models in AMIP4K and 10 models in AMIPFuture and AMIP4 $\times \mathrm{CO}_{2}$ ) agree on the sign of change. 
subtropical winds, which is a robust dynamical response to the global warming and is linked, via the thermal wind relation, to the warming amplification in the upper tropical troposphere.

The atmospheric response in the AMIP $4 \times \mathrm{CO}_{2}$ experiment includes strong radiative cooling in the stratosphere, due to increases in $\mathrm{CO}_{2}$, which exceeds $10 \mathrm{~K}$ at $10 \mathrm{hPa}$, but it has only a weak tropospheric warming with values less than $1 \mathrm{~K}$ everywhere except in the lower polar troposphere. In contrast to the other two experiments, the polar night jet becomes stronger and there is no strengthening of subtropical winds in this experiment, which is due to the lack of warming amplification in the upper tropical troposphere.

We next demonstrate that the dipole pattern in the stratospheric temperatures in the AMIP4K and AMIPFuture experiments is robust across the models. As was noted above the air temperature changes contain both dynamical and radiative components. While GHG concentrations in these experiments remain the same as in AMIP, the radiative temperature changes in the stratosphere may be caused, for example, by changes in stratospheric water vapor concentrations, which directly depend on tropical tropopause temperatures and therefore increases in these simulations. To separate the dynamical changes we adopt the methodology by Ueyama and Wallace (2010) and remove global mean temperature change for each month, pressure level, and model. The assumption here is that the radiative effects on temperature changes are latitudinally independent. Although the radiative effects may be latitude dependent and thus not fully removed by this procedure, we assume that it is sufficient to highlight the effect of the dynamics. Indeed, if the latitudinally dependent radiative component due to the stratospheric water vapor was dominant in the residual field then it would show a maximum residual cooling in high latitudes (Forster and Shine 2002), which is the opposite of the pattern we show below. Figure 2 shows temperature changes at $20 \mathrm{hPa}$ for individual models after the global mean temperatures $T^{*}$ are removed. We choose $20 \mathrm{hPa}$ because this level is high enough to be mainly affected by the deep branch of the BD circulation. For most of the models in AMIP4K and AMIPFuture the global mean temperature change at this level is less than $1 \mathrm{~K}$; thus its removal has only minor impact on the result. The two exceptions are CNRM-CM5 and MRI-CGCM3, which have global mean cooling of about $3 \mathrm{~K}$ and warming of $1 \mathrm{~K}$, respectively. Figure 2 shows that the dipole pattern with anomalous warming of the polar stratosphere and cooling of the tropical stratosphere is indeed robust across individual models in AMIP4K and AMIPFuture. Interpreting the temperature dipole as the signature of the BD changes, a robust strengthening of the deep branch of the BD circulation can be inferred. The analysis of the seasonal cycle of $T^{*}$ changes in AMIP4K and AMIPFuture across individual models (see Fig. S1 in the supplemental material for the AMIP4K case) further demonstrates that in all models the polar warming maximizes in winter months in conjunction with maximum in tropical cooling.

While most of the models simulate maximum warming at the pole, a few models, namely MIROC5 in all simulations and HadGEM2-A in AMIPFuture, suggest maximum warming around $60^{\circ} \mathrm{N}$. Through the thermal wind equation such a pattern is consistent with strengthening of the winds north of $60^{\circ} \mathrm{N}$. Indeed, MIROC5 is the only model that simulates strengthening of the winds through the polar stratosphere in both AMIP4K and AMIPFuture, while HadGEM2-A is the only other model that simulates strengthening of the polar winds in AMIPFuture.

Comparing AMIP4K and AMIPFuture one can note a tendency for the same models to consistently simulate relatively large or small warming in both experiments. For example, the largest polar $T^{*}$ change in both AMIP4K and AMIPFuture are simulated by the two MPI models, while BCC_CSM1.1, CCSM4, HadGEM2-A, and MIROC5 have small warming in both experiments. Internal variability is known to contribute to future circulation changes (Butchart et al. 2000; Shepherd 2014); however, the lack of ensemble simulations limits our ability to estimate the contribution of the internal variability in these experiments. IPSL-CM5A-LR is the only model that provides two simulations that differ by initial conditions only and thus can be used to assess the role of the internal variability. The difference between their simulated changes in the Arctic stratosphere is about $1 \mathrm{~K}$ (Fig. 2). While it does not suggest a dominant role of the internal variability in comparison to the model uncertainty (i.e., intermodel spread), we emphasize that the number of ensemble simulations is not sufficient to reach a decisive conclusion.

The $T^{*}$ changes in the AMIP $4 \times \mathrm{CO}_{2}$ experiment are not robust across the models and, in general, weaker than those in the other two experiments, although some models (the IPSL models, HadGEM2-A, and MRI-CGCM3) simulate dynamical polar cooling of 2-6 K (Fig. 2c), which dominates the multimodel mean response and contributes to the strengthening of the polar night jet seen in Fig. 1 .

To summarize, we have shown that there is a robust warming in the Arctic stratosphere in the CMIP5 AMIP4K and AMIPFuture experiments, which we interpret as an indication of a strengthening of the deep 
a) amip $4 \mathrm{~K}, \mathrm{~T}^{*}, 20 \mathrm{hPa}$

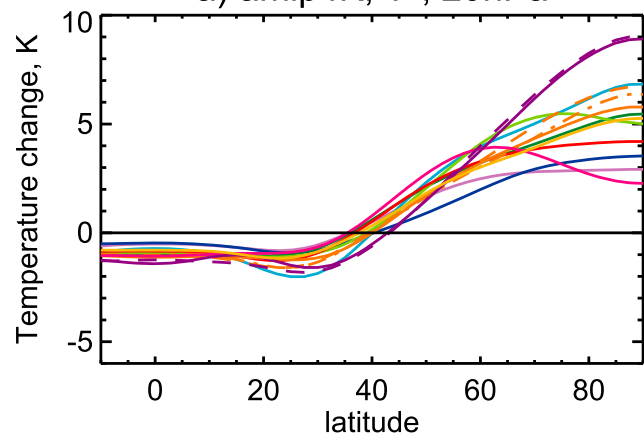

c) amip $4 \times C O 2, \mathrm{~T}^{*}, 20 \mathrm{hPa}$

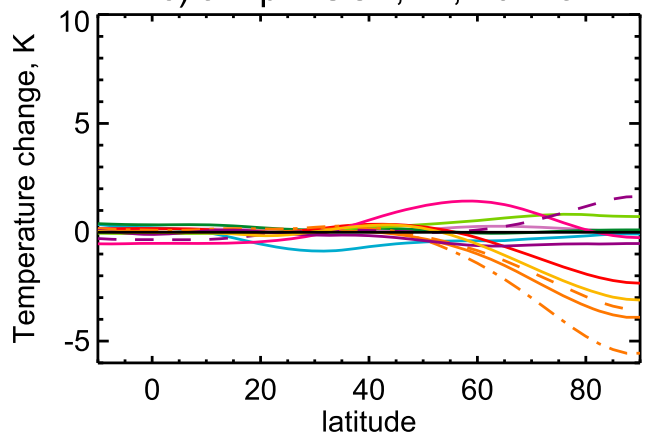

b) amipFuture, $\mathrm{T}^{*}, 20 \mathrm{hPa}$

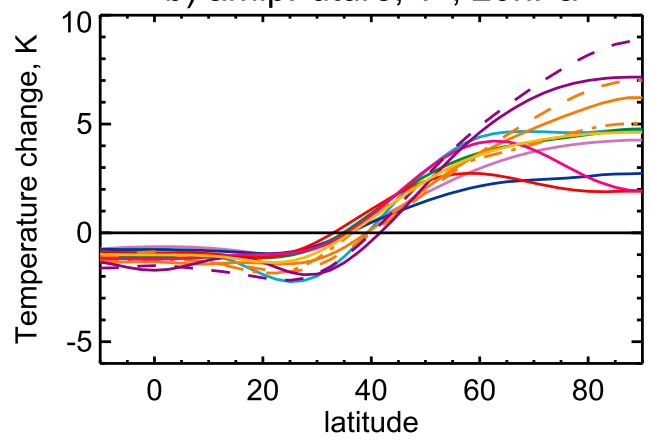

d) EOF1, T@20hPa

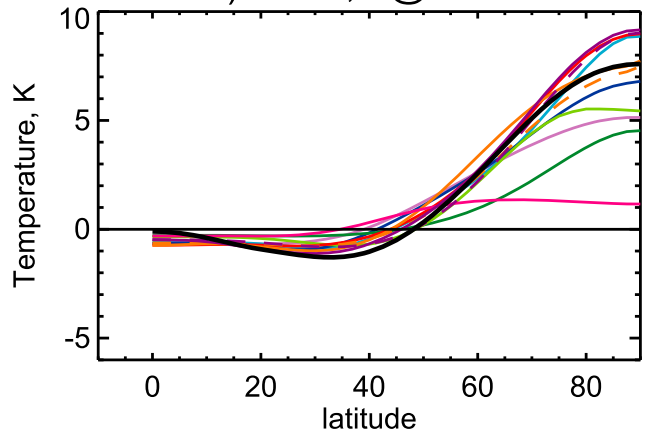

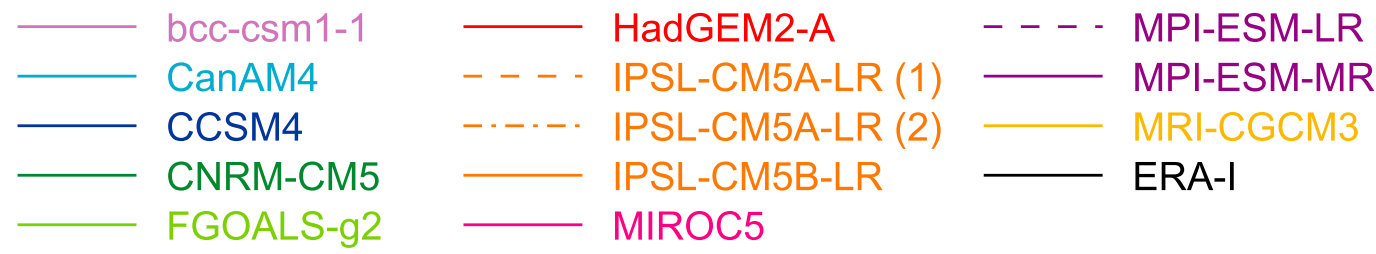

FIG. 2. (a)-(c) Difference in DJF zonal mean temperatures at $20 \mathrm{hPa}$ between future and past AMIP experiments for individual models with global mean temperature change removed for each model. (d) The first EOF of the DJF monthly mean $T^{*}$ at $20 \mathrm{hPa}$ in the AMIP experiment (color) and ERA-I (black).

branch of the BD circulation. In the following sections, we test the idea that changes in stationary waves provide for a plausible mechanism for the documented warming and its intermodel spread.

\section{b. Changes in stratospheric meridional eddy heat flux}

In this section we consider changes in the stratospheric drivers of the BD circulation. We focus on changes in the eddy heat flux entering the stratosphere at $100 \mathrm{hPa}$, which is a widely accepted proxy of the tropospheric forcing. As will be shown below this approach can account for a significant fraction of changes in the stratospheric temperatures in these simulations. To account for changes in wave propagation and absorption within the stratosphere, however, would require higherresolution data in both space (more vertical levels) and time (daily output frequency) than that available for most of the models in this study and is therefore not included here. Figure 3 a shows the annual cycle in the response of the heat flux in the AMIP4K experiment averaged across the six models (CNRMCM5, FGOALS-g2, HadGEM2-A, IPSL-CM5A-LR, IPSL-CM5B-LR, and MIROC5) for which daily data, needed to calculate the total heat flux, are available. All six models simulate an increase in the heat flux at midlatitudes maximizing in winter, which is expected to strengthen BD circulation and warm the polar stratosphere. A decrease of the heat flux north of $65^{\circ} \mathrm{N}$, noticed earlier by McLandress and Shepherd (2009) in their model, can also be seen. Interestingly, the weaker heat flux changes in summer and autumn are also consistent across the models as well as changes in the tropics.

It is possible to separate the total heat flux into contributions from slowly varying quasi-stationary waves based on monthly mean fields and the residual flux due 
(a) MMM, total

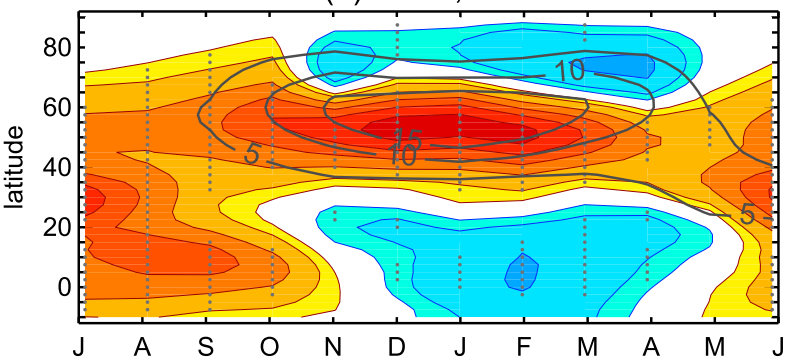

(b) MMM, stationary

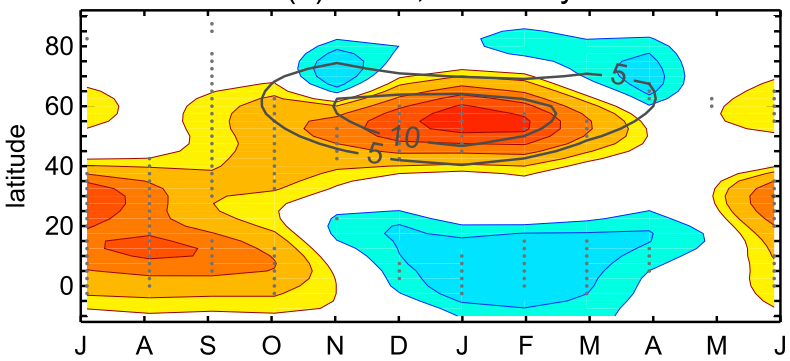

(c) MMM, transient
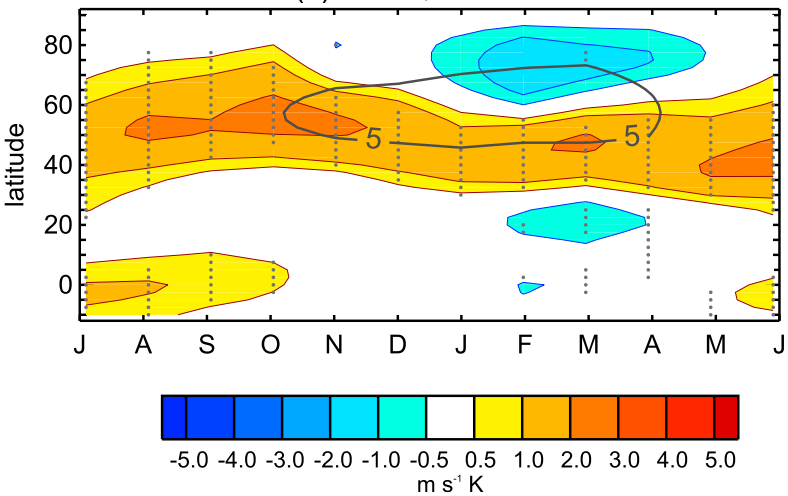

FIG. 3. Multimodel mean changes in the heat flux at $100 \mathrm{hPa}$ between AMIP and AMIP4K for the six models (CNRM-CM5, FGOALS-g2, HadGEM2-A, IPSL-CM5A-LR, IPSL-CM5B-LR, and MIROC5) with available daily data. The gray contours show climatological mean heat flux $\left(\mathrm{m} \mathrm{s}^{-1} \mathrm{~K}\right)$ in the AMIP experiment. Gray dots indicate grid points where all six models agree on the sign of change.

to the transient waves. These are shown in Figs. $3 \mathrm{~b}$ and $3 \mathrm{c}$, respectively. The winter maximum in the heat flux increase is dominated by the component due to the quasistationary waves, which accounts for $40 \%-60 \%$ in November-December and for more than $80 \%$ in January-February of the total heat flux increase between $45^{\circ}$ and $70^{\circ} \mathrm{N}$. The contribution of transient waves is significant during all seasons and has a broad maximum from August to November. The latitude of the maximum transient heat flux increase moves from $50^{\circ}-$ $60^{\circ} \mathrm{N}$ during summer-autumn to a more southern position $\left(40^{\circ}-50^{\circ} \mathrm{N}\right)$ during winter and so follows the annual cycle of the position of the eddy-driven jet. a) amip4K

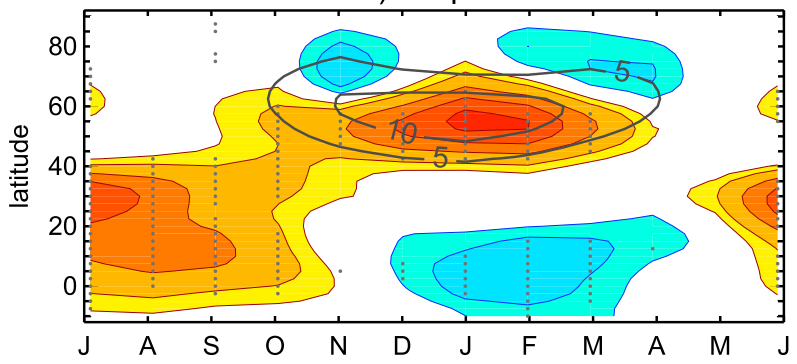

b) amipFuture
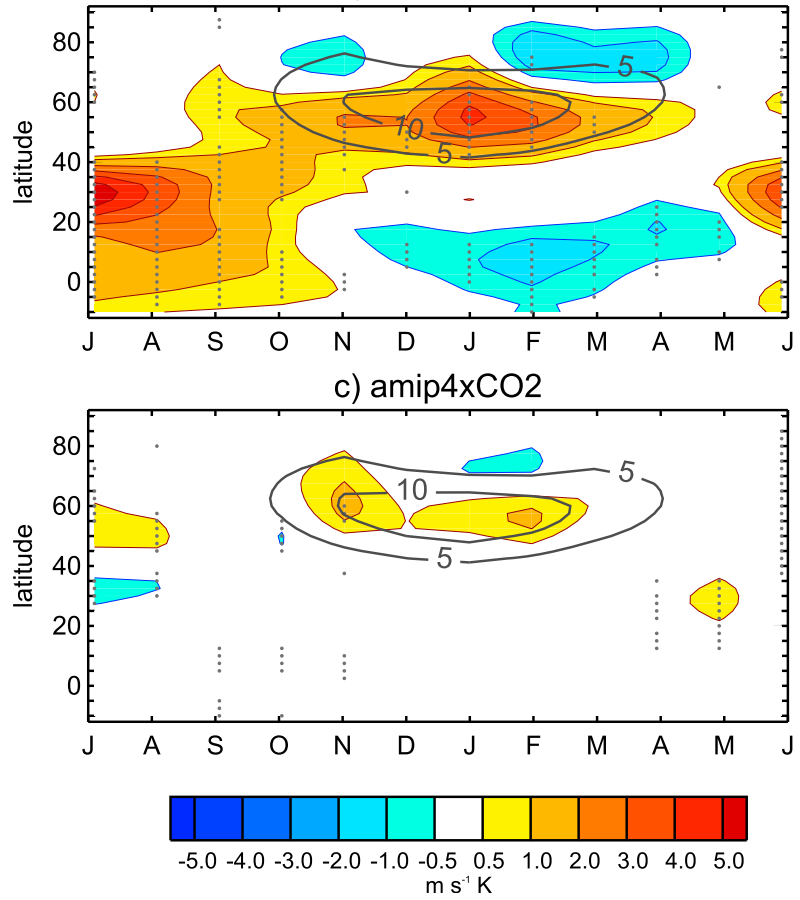

FIG. 4. Changes in the stationary heat flux at $100 \mathrm{hPa}$ in the AMIP4K, AMIPFuture, and AMIP $4 \times \mathrm{CO}_{2}$ experiments based on all models (12 models in AMIP4K and 11 models in AMIPFuture and AMIP4 $\times \mathrm{CO}_{2}$ ). Gray contours show climatological mean heat flux $\left(\mathrm{m} \mathrm{s}^{-1} \mathrm{~K}\right)$ in the AMIP experiment. Gray dots indicate grid points where more than $90 \%$ of the models (i.e., at least 11 models in AMIP4K and 10 models in AMIPFuture and AMIP4 $\times \mathrm{CO}_{2}$ ) agree on the sign of change.

Figure 4 shows the component of the heat flux increase due to the quasi-stationary waves calculated from monthly mean data for all 12 models and for the three global warming scenarios. Figure S2 shows the changes for individual models in AMIP4K. Again, a remarkable similarity across individual models as well as between AMIP4K and AMIPFuture can be noticed. On the other hand, there is little consistency in heat flux changes between the models in AMIP4 $\times \mathrm{CO}_{2}$.

Figures 3 and 4 show that there is a robust increase in the wave activity flux into the stratosphere in AMIP4K and AMIPFuture, which can explain the strengthening 
TABLE 2. Correlation and regression coefficients between total eddy heat flux at $100 \mathrm{hPa}, 45^{\circ}-70^{\circ} \mathrm{N}$, averaged from $1 \mathrm{Nov}$ to $15 \mathrm{Feb}$ and the DJF mean polar $\left(60^{\circ}-90^{\circ} \mathrm{N}\right)$ temperature at $20 \mathrm{hPa}$. Also shown are the fractions of the DJF polar stratospheric warming between the AMIP and AMIP4K experiments attributable to the increase in the eddy heat flux.

\begin{tabular}{lccccr}
\hline \hline \multirow{2}{*}{ Model } & \multicolumn{2}{c}{ Correlation coef } & & Regression coef, \\
\cline { 2 - 5 } & AMIP & AMIP4K & & $\begin{array}{c}\text { Fraction due to total } \\
\text { heat flux increase (\%) }\end{array}$ & $\begin{array}{c}\text { Fraction due to stationary } \\
\text { heat flux }(\%)\end{array}$ \\
\hline CNRM-CM5 & 0.71 & 0.55 & 0.78 & 56 & 41 \\
FGOALS-g2 & 0.89 & 0.61 & 1.52 & 89 & 64 \\
HadGEM2-A & 0.85 & 0.86 & 1.47 & 148 & 102 \\
IPSL-CM5A-LR & 0.59 & 0.71 & 0.89 & 98 & 74 \\
IPSL-CM5B-LR & 0.76 & 0.71 & 1.39 & 112 & 101 \\
MIROC5 & 0.61 & 0.62 & 0.75 & 61 & 29 \\
\hline
\end{tabular}

of the BD circulation and dynamical warming of the polar stratosphere. We will next use more quantitative estimations to demonstrate that this increase can explain a considerable fraction of the stratospheric warming seen in Figs. 1 and 2. For this purpose we first establish the link between the eddy heat flux and polar stratospheric temperatures in the AMIP experiment by the means of a linear regression. Specifically we regress 20-hPa monthly temperature anomalies with respect to the annual cycle averaged over December-February and over the polar cap $\left(60^{\circ}-90^{\circ} \mathrm{N}\right)$ onto the total eddy heat flux at $100 \mathrm{hPa}$ averaged between $45^{\circ}$ and $70^{\circ} \mathrm{N}$ and between 1 November and 15 February. The lag between the heat flux and the temperatures is chosen to account for the cumulative effect of the heat flux on the temperatures (Newman et al. 2001). The fraction $\delta$ of the warming (percent) attributable to the increase in the heat flux between present and future experiments can then be estimated (e.g., Thompson et al. 2000) as follows:

$$
\delta=100 \times b \frac{\overline{\mathrm{HF}}_{f}-\overline{\mathrm{HF}}_{p}}{\bar{T}_{f}-\bar{T}_{p}},
$$

where $b$ is the slope parameter of the regression in the AMIP experiment; HF is the $100-\mathrm{hPa}$ heat flux; $T$ is the 20-hPa air temperature anomalies; $p$ and $f$ indices indicate present (AMIP) and future (AMIP4K) experiments, respectively; and the overbar indicates mean value over the experiments. As in section 3 a the global mean temperature change between AMIP and AMIP4K is removed in order to account for possible radiative changes. (This procedure has considerable effect only on CNRM-CM5, which has global cooling of about $3 \mathrm{~K}$.) Since it is the total heat flux that influences the temperatures, the calculations are only done for the six models with the daily data.

The results of the calculations for each model for AMIP4K, as well as the correlation coefficients between the heat flux and the polar stratospheric temperatures, are shown in Table 2. The correlation coefficient between the heat flux and the winter stratospheric temperatures at $20 \mathrm{hPa}$ in the AMIP experiment varies from 0.59 to 0.89 depending on the model. (Table 2 also shows that the relation changes somewhat between AMIP and AMIP4K but remains statistically significant for all models.) In all the models, more than half of the stratospheric warming between AMIP and AMIP4K can be attributed to the increase in the total heat flux. By replacing the total heat flux by its quasi-stationary component in Eq. (1), we find that in four out of six models only the increase in the quasi-stationary component can account for more than half of the warming. Interestingly, the results for HadGEM2-A and IPSL-CM5B-LR suggest that the heat flux increase can account for more than $100 \%$ of the warming. This may be due to the uncertainty inherited in the approach but may also indicate that some other mechanisms (e.g., changes in gravity waves) reduce the warming due to the planetary wave forcing.

To shed more light on the mechanism of dynamical changes we calculate the separate contribution of different waves to the increase in the heat flux. The results for AMIP4K during the November-February (NDJF) season are summarized in Table 3 . It is seen that the wavenumber 1 is responsible for the eddy heat flux increase in all models. In some models negative changes due to wavenumber 2 somewhat offset the increase due to wavenumber 1 . The other waves play only a minor role.

To summarize, we find that the wave activity flux from the troposphere to the stratosphere in midlatitudes increases in all models in the AMIP4K and AMIPFuture experiments but not in AMIP4 $\times \mathrm{CO}_{2}$. The increase provides an explanation for the dynamical warming of the Arctic stratosphere. In winter the increase is dominated by the quasi-stationary wave flux, which, in turn, is dominated by the wavenumber 1 component. Simple estimations suggest that the increase of the wave activity flux can account for more than half of the polar stratospheric warming simulated by the models. 
TABLE 3. Changes in NDJF mean stationary eddy heat flux $\left(\mathrm{m} \mathrm{s}^{-1} \mathrm{~K}\right)$ between AMIP and AMIP4K: total flux and flux due to zonal wavenumbers 1 and 2 , averaged over $45^{\circ}-70^{\circ} \mathrm{N}$.

\begin{tabular}{|c|c|c|c|c|}
\hline Model & Total & Wavenumber 1 & Wavenumber 2 & Residual \\
\hline BCC_CSM1.1 & 1.83 & 1.46 & 0.10 & 0.26 \\
\hline CanAM4 & 0.89 & 1.80 & -0.74 & -0.18 \\
\hline CCSM4 & 1.14 & 1.69 & -0.55 & 0.00 \\
\hline CNRM-CM5 & 2.17 & 3.39 & -1.25 & 0.03 \\
\hline FGOALS-g2 & 2.25 & 2.52 & -0.14 & -0.13 \\
\hline HadGEM2-A & 2.41 & 4.93 & -2.71 & 0.19 \\
\hline IPSL-CM5A-LR & 4.02 & 4.57 & -1.00 & 0.44 \\
\hline IPSL-CM5B-LR & 2.98 & 3.83 & -0.58 & -0.27 \\
\hline MIROC5 & 1.38 & 1.74 & -0.25 & -0.11 \\
\hline MPI-ESM-LR & 1.67 & 2.65 & -0.87 & -0.12 \\
\hline MPI-ESM-MR & 2.10 & 2.76 & -0.80 & 0.14 \\
\hline MRI-CGCM3 & 3.81 & 4.34 & -0.70 & 0.18 \\
\hline
\end{tabular}

\section{c. Changes in tropospheric stationary waves}

To illustrate the changes in tropospheric waves, we use the eddy geopotential height anomaly $Z^{\prime}=Z-\bar{Z}$ (where $Z$ is the geopotential height and the overbar indicates zonal average) as a measure of wave disturbances. Figure 5 shows the multimodel NDJF mean change in stationary geopotential height anomaly $Z^{\prime}$ at $500 \mathrm{hPa}$ between AMIP and the climate change experiments. Figure $\mathrm{S} 3$ shows the change in $Z^{\prime}$ at $500 \mathrm{hPa}$ for individual models in AMIP4K. Once again a remarkable consistency of the changes across the models can be noted in AMIP4K and AMIPFuture. The main part of the response is a wave train propagating from East Asia across the Pacific and North America toward Eurasia, given that the largest anomalies are the low in the eastern Pacific and the high over Canada. The analysis of the vertical structure shows that this anomalous pattern is equivalently barotropic between 700 and $200 \mathrm{hPa}$ (not shown). The $Z^{\prime}$ changes in AMIP $4 \times \mathrm{CO}_{2}$ are weak and inconsistent with the two other experiments, as was found in the other diagnostics.

We next show that the pattern of anomalies in Figs. 5a and $5 \mathrm{~b}$ is consistent with enhanced wave flux into the stratosphere and with a polar stratosphere warming. Previously, tropospheric precursors of Arctic stratospheric vortex anomalies have been studied by Orsolini et al. (2009) and Garfinkel et al. (2010). In particular, Garfinkel et al. (2010) applied a correlation analysis to daily data using a 10-day lag between $Z^{\prime}$ and a stratospheric index. Here we use monthly data available for all models. Specifically, we regress December, January, and February monthly polar cap $\left(60^{\circ}-90^{\circ} \mathrm{N}\right) \mathrm{T}$ at $20 \mathrm{hPa}$ on the November, December, and January monthly $Z^{\prime}$ at each grid point in models as well as in ERA-Interim (hereafter ERA-I). To demonstrate that the link between tropospheric waves and the polar stratosphere a) amip4K

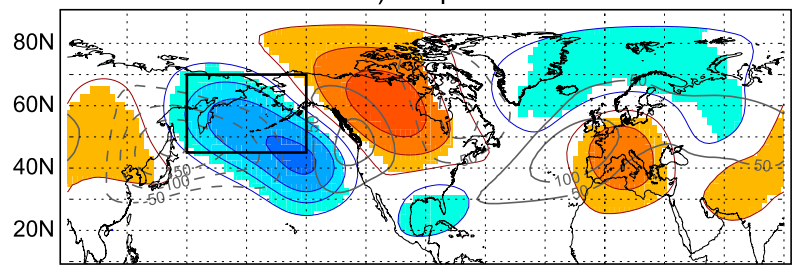

b) amipFuture

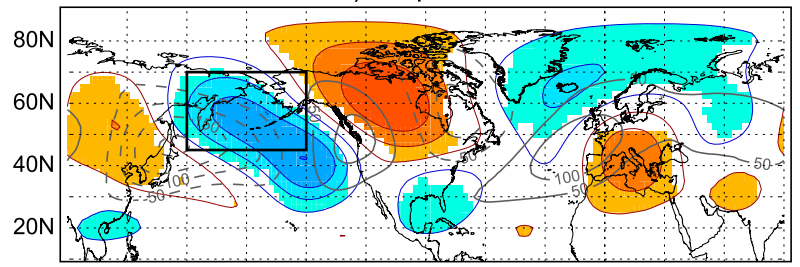

c) $\operatorname{amip} 4 \times \mathrm{CO} 2$

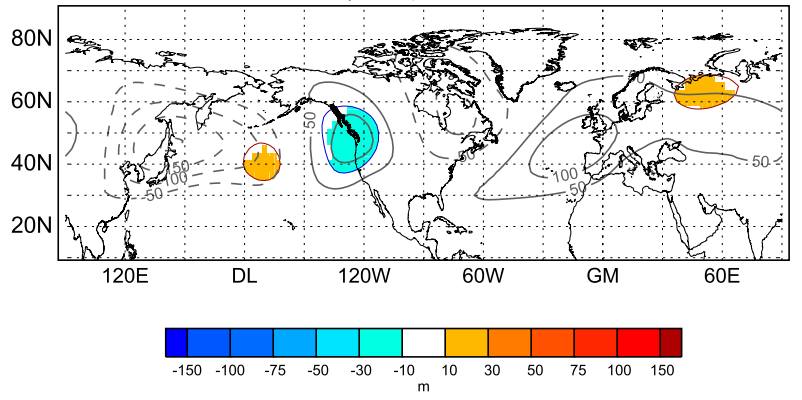

FIG. 5. Changes in the NDJF eddy geopotential height anomaly (m) at $500 \mathrm{hPa}$. Only areas where more than $90 \%$ of the models (i.e., at least 11 models in AMIP4K and 10 models in AMIPFuture or AMIP $4 \times \mathrm{CO}_{2}$ ) agree on the sign of change are shaded. Gray contours show climatological mean $Z^{\prime}$. The black rectangles in (a) and (b) mark the area used to calculate the $Z^{\prime}$ index for section $3 \mathrm{e}$.

involves increased wavenumber 1 flux, as suggested by the analysis in section $3 \mathrm{~b}$, we also regress November, December, and January monthly wavenumber 1 heat flux at $100 \mathrm{hPa}$, averaged over $45^{\circ}-70^{\circ} \mathrm{N}$, on the November, December, and January monthly $Z^{\prime}$ at each grid point. A one-month lag between stratospheric temperatures and $Z^{\prime}$ at $500 \mathrm{hPa}$ is introduced to focus on oneway (upward) causality. No lag is introduced between $100 \mathrm{hPa}$ heat flux and $Z^{\prime}$ at $500 \mathrm{hPa}$. We tested that removing trends prior to calculating regressions has a negligible impact on the results. Here, the results for not detrended data are presented.

The regression patterns are shown in Fig. 6. The results for ERA-I (Fig. 6a) show considerable similarity with those by Garfinkel et al. (2010, see their Fig. 1). Both patterns include a low over the Bering Strait, a high south of the Hudson Bay, and a high over northeastern Europe. The anomalies over the North Pacific were also found by Orsolini et al. (2009), who showed that cold 
a) Regression T@20hPa, ERA-I

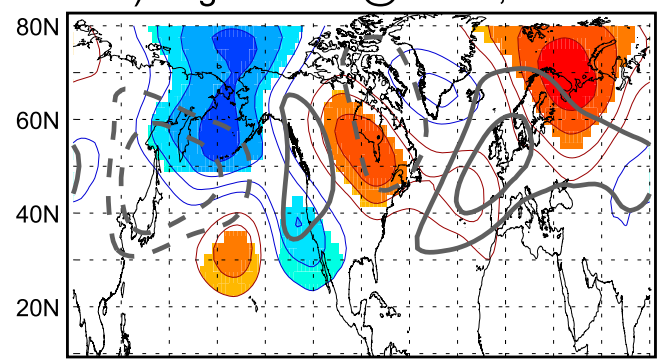

c) Regression T@20hPa, amip (0.71)
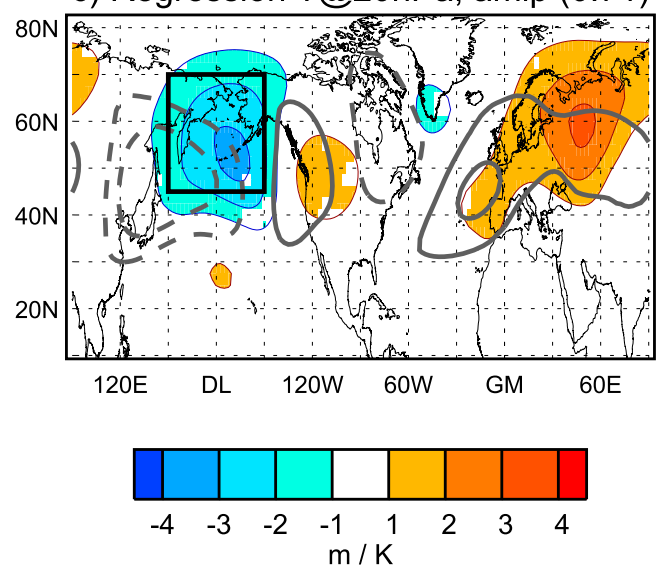

b) Regression vtw1@100hPa, ERA-I

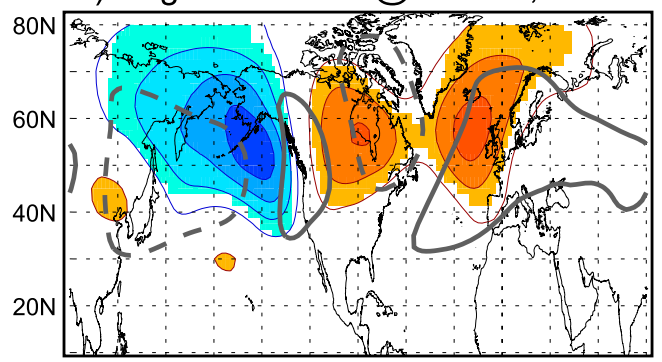

d) Regression vtw1@100hPa, amip (0.94)
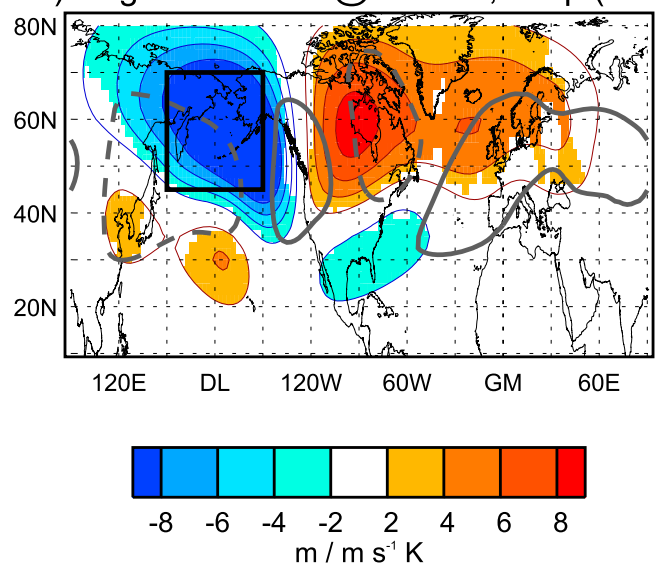

FIG. 6. Regressions of monthly $Z^{\prime}$ (November, December, and January) on (left) monthly temperature (December, January, and February) at $20 \mathrm{hPa}$ averaged over $60^{\circ}-90^{\circ} \mathrm{N}$ and on (right) monthly heat flux (November, December, and January) due to quasi-stationary wavenumber 1 at $100 \mathrm{hPa}$. The results are for (a),(b) ERA-I and (c),(d) multimodel ensemble mean based on the 12 models in the AMIP experiment. Gray contours show climatological mean positions of \pm 60 - and 120 -m contours of $Z^{\prime}$ (negative values are dashed). The black rectangles in (c) and (d) mark the area used to calculate the $Z^{\prime}$ index for section 3e. Only areas where (a),(b) regression coefficients are significant at $p=0.05$, (c) more than $66 \%$ of the models (i.e., at least 8 models) agree on the sign of regression coefficient, or (d) more than $90 \%$ of the models (i.e., at least 11 models) agree on the sign of regression coefficient are shaded. Numbers in the parentheses in (c) and (d) indicate pattern correlation coefficients between corresponding regression patterns in ERA-I and multimodel ensemble mean.

stratospheric months are preceded by a high over the North Pacific, although in their case the maximum correlation is shifted westward toward eastern Siberia. Figure $6 \mathrm{~b}$ shows that the low over the North Pacific also corresponds to an increased wavenumber 1 heat flux to the stratosphere.

The multimodel mean patterns, calculated as an average over individual model regression patterns, are shown in Figs. 6c and 6d for the regression on stratospheric temperatures (Fig. 6c) and wavenumber 1 eddy heat flux at $100 \mathrm{hPa}$ (Fig. 6d). The regressions of $Z^{\prime}$ on stratospheric temperatures for individual models are shown in Fig. S4. The multimodel patterns are very similar to those from the reanalysis. The important result is that an anomalous low in the northeastern Pacific and an anomalous high over Canada seen in the pattern of changes in Fig. 5 strongly project on the regression pattern, while the changes over the North Atlantic and northern Europe do not, suggesting that the warming of the polar stratosphere in the future climate experiments is related to the geopotential height changes over the Pacific and Canada.

Not all the models reproduce the same regression pattern of stratospheric temperatures on $Z^{\prime}$ as seen in the multimodel mean in Fig. 6c (see Fig. S4). At the same time the link between tropospheric disturbances and the heat flux at $100 \mathrm{hPa}$, which involves no lag, is robust across all models, which suggests that the same mechanism is at work in all models. In particular, BCC_CSM1.1 and HadGEM2-A results suggest that a negative monthly $Z^{\prime}$ over the northeastern Pacific corresponds to an increased wavenumber 1 flux into the stratosphere, consistent with other models and ERA-I; however, a positive monthly $Z^{\prime}$ in the same region corresponds to a positive anomaly in polar stratosphere temperatures one month later, contrary to the ERA-I 
a) amip4K

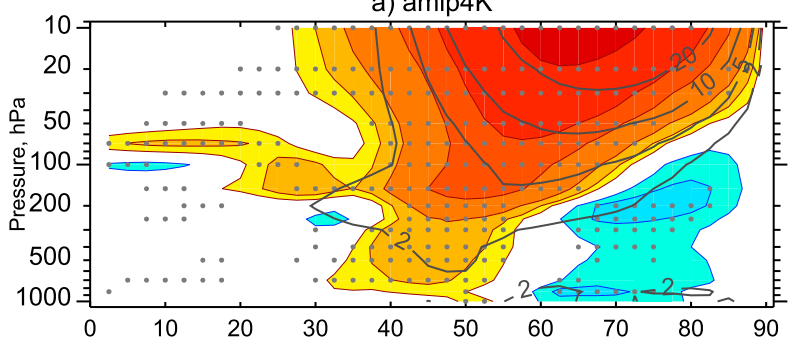

b) amipFuture

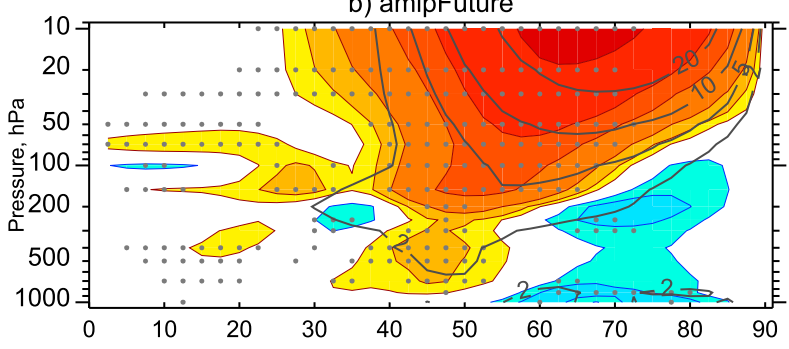

c) amip $4 x \mathrm{CO} 2$

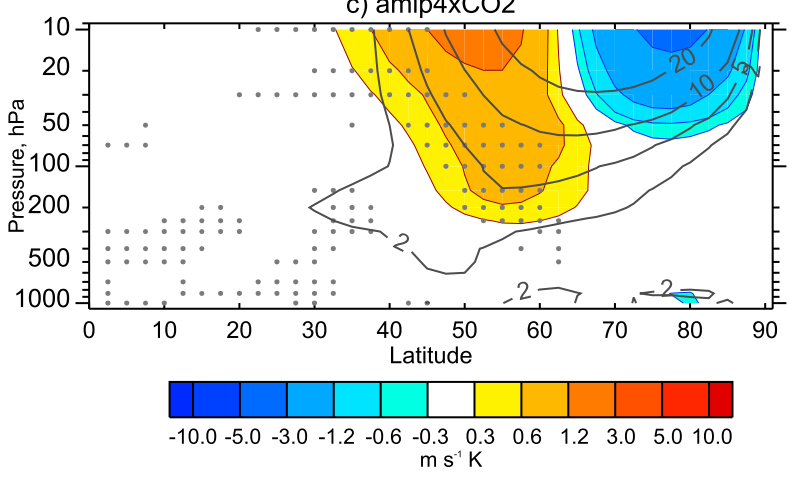

FIG. 7. Multimodel mean changes in the NDJF mean heat flux due to quasi-stationary wavenumber 1 in the AMIP4K, AMIPFuture, and AMIP $4 \times \mathrm{CO}_{2}$ experiments based on all models (12 models in AMIP4K and 11 models in AMIPFuture and AMIP4 $\times \mathrm{CO}_{2}$ ). The gray contours show climatological mean heat flux $\left(\mathrm{m} \mathrm{s}^{-1} \mathrm{~K}\right)$ in the AMIP experiment. Gray dots indicate grid points where more than $90 \%$ of the models (i.e., at least 11 models in AMIP4K and 10 models in AMIPFuture and AMIP4 $\times \mathrm{CO}_{2}$ ) agree on the sign of change.

and multimodel mean results. We suggest that in these models the one-month lag is not optimal to untangle the upward influence that takes place on shorter time scales. Thus, submonthly lags are needed. To test whether this is indeed the case we use daily data for the six models for which they are available (see Table 2) and, following Garfinkel et al. (2010), correlate $Z^{\prime}$ at each grid point with 10-day stratospheric polar temperature tendencies for each day during the DJF seasons. The results (not shown) reveal similar patterns as those in Fig. 6c with significant negative correlation coefficients over the Bering Strait and the northeastern Pacific for all six models, including HadGEM2-A. This result supports our conclusion that an anomalous low over the North Pacific is consistent with a stratospheric warming.
Enhanced propagation of wave activity due to wavenumber 1 into the stratosphere was documented in section $3 \mathrm{~b}$. We next more firmly link stratospheric wave driving to the changes in the tropospheric waves. Figure 7 shows a vertical cross section of NDJF mean heat flux due to wavenumber 1 , to diagnose upward propagation of wave activity throughout the atmosphere. Figure S5 shows the wavenumber 1 heat flux for individual models in the AMIP4K experiments. Enhanced vertical propagation of wavenumber 1 activity is seen in the troposphere in the AMIP4K and AMIPFuture experiments, peaking between $40^{\circ}$ and $50^{\circ} \mathrm{N}$, and is robust across individual models. Although wave refraction can contribute to these changes (especially in the upper troposphere), integrated eddy heat flux across latitudes is positive throughout most of the troposphere suggesting increased generation of wave activity. Similar figures for wavenumbers 2 and 3 also show an enhanced heat flux in the high-latitude troposphere north of $50^{\circ} \mathrm{N}$; however, in those cases the enhancement is confined within the troposphere (not shown), which is consistent with the lack of stratospheric forcing from these waves as documented in section $3 \mathrm{~b}$. We note that, while the increase in the tropospheric heat flux due to wavenumber 1 is robust in the AMIP simulations, previous single model studies reported a lack of increased wave generation (e.g., Sigmond et al. 2004; Garcia and Randel 2008). Further progress on the generality of our results therefore requires better understanding of the mechanisms for the increased propagation of the planetary wave activity from the troposphere.

So far we have presented evidence that the response of tropospheric waves to global warming is expected to increase wavenumber 1 flux into the stratosphere and to warm the polar stratosphere. But we still lack an understanding of the causes of changes in the tropospheric waves seen in Figs. 5 and 7. Stationary planetary waves are generated in the troposphere by interaction of the mean flow with orography, by diabatic heating, and by nonlinear interactions between transient waves. Recently, Simpson et al. (2016) demonstrated a dominant role for changes in the zonal mean basic state in producing the intermediate-scale stationary wave response to climate change. However, the investigation and the assessment of the relative importance of all sources of changes in the largest (wavenumber $<3$ ) stationary waves would require a work by its own. Here we therefore restrict ourselves only to the search for evidence that the increased upward propagation of wavenumber 1 is related to the strengthening of the zonal winds in the upper troposphere. Simpson et al. (2016) studied the eastward shift of the meridional wind patterns at $20^{\circ}-40^{\circ} \mathrm{N}$ in a climate change scenario and 
showed that the simulated changes in stationary wave patterns can be understood as a wave lengthening, in a response to strengthening of the zonal winds in the upper troposphere. Although they focused on the response in lower latitudes and higher zonal wavenumbers, Figs. 2 and 3 in Simpson et al. (2016) are remarkably consistent with the anomalies in our Fig. 5, in particular the anomalies in the North Pacific discussed above. It is therefore of interest to ask if such a lengthening could occur for the largest planetary waves.

Figure 8 shows the zonal wavenumber amplitude spectrum change at $500 \mathrm{hPa}$. Focusing on AMIP4K and AMIPFuture and on the changes in the longest waves that can propagate to the stratosphere, Figs. $8 \mathrm{a}$ and $8 \mathrm{~b}$ show an increase in the amplitude of wavenumber 1 between $40^{\circ}$ and $55^{\circ} \mathrm{N}$. The increase in wavenumber 1 coincides with its climatological maximum. Between $40^{\circ}$ and $50^{\circ} \mathrm{N}$, we note as well an amplitude decrease for wavenumbers 2 and 3, which can be interpreted as a wave-lengthening effect (i.e., favoring the longest wavenumber 1), although a wave refraction toward higher latitudes can also contribute to the weakening of these waves in midlatitudes. Between $50^{\circ}$ and $60^{\circ} \mathrm{N}$, the amplitudes increase for all waves, a change more consistent with increased wave generation. Given that wave generation by orography is proportional to the wind speed (i.e., Held 1983; Held et al. 2002), it is plausible that strengthened zonal winds in the subtropics and midlatitudes (Fig. 1), where important wave sources, such as Tibet, are located, lead to increased planetary wave generation. The change is then visible at higher latitudes, as the waves propagate poleward from their source region.

\section{d. Intermodel spread in stratospheric response}

In this section, we first assess the intermodel spread in the tropospheric and stratospheric circulation response to test the links discussed in section 3c. We then ask if the difference among the stratospheric responses can be explained by biases in stratospheric variability.

According to the discussion in the previous section, the anomalous low in the North Pacific is consistent with increased amplitude and larger wavenumber 1 heat flux to the stratosphere and warmer Arctic stratosphere; thus a larger negative $Z^{\prime}$ anomaly in the North Pacific could lead to a larger stratospheric warming. Figures 9a and $9 \mathrm{~b}$ show that this is indeed the case. The intermodel spread in $Z^{\prime}$ response averaged over $45^{\circ}-70^{\circ} \mathrm{N}$ and $150^{\circ} \mathrm{E}-150^{\circ} \mathrm{W}$ (see Figs. 5 and 6 for the position of the area) can explain more than half $\left(r^{2}=0.63\right)$ of the intermodel spread in the stratospheric warming in AMIP4K. The link is weaker in AMIPFuture $\left(r^{2}=\right.$ 0.31 ), mainly because of one of the two IPSL-CM5A-LR

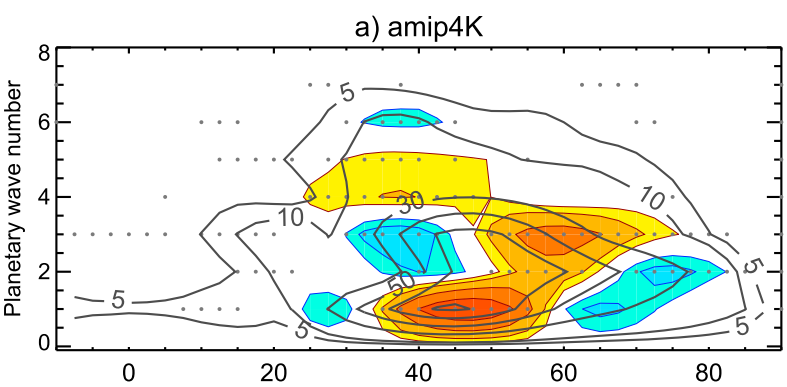

b) amipFuture

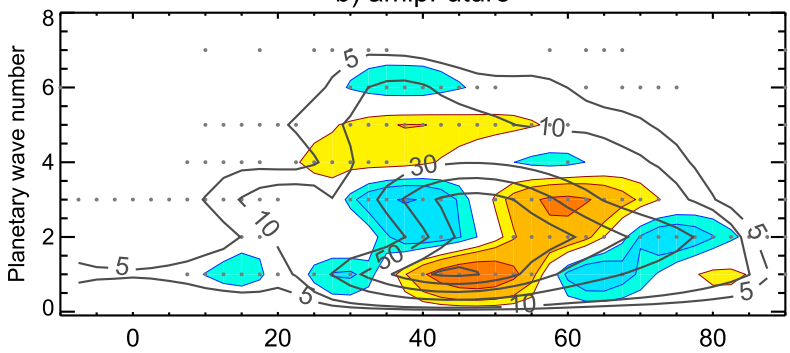

c) amip $4 x \mathrm{CO} 2$

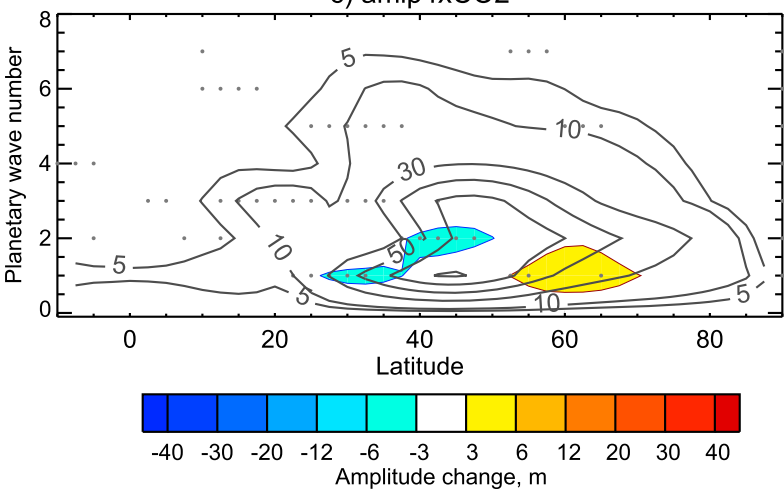

FIG. 8. Multimodel mean changes in the amplitude of NDJF eddy geopotential height anomaly $(\mathrm{m})$ at $500 \mathrm{hPa}$ as function of zonal wavenumber in the AMIP4K, AMIPFuture, and AMIP $4 \times \mathrm{CO}_{2}$ experiments based on all models (12 models in AMIP4K and 11 models in AMIPFuture and AMIP4 $\left.\times \mathrm{CO}_{2}\right)$. The gray contours show climatological mean amplitudes $(\mathrm{m})$ in the AMIP experiment. Gray dots indicate grid points where more than $90 \%$ of the models (i.e., at least 11 models in AMIP4K and 10 models in AMIPFuture and AMIP $4 \times \mathrm{CO}_{2}$ ) agree on the sign of change.

runs, which is a large outlier with a strongly negative $Z^{\prime}$ anomaly. Excluding this run would result in a substantially stronger correlation $(r=-0.69)$ with almost half of the variance explained $\left(r^{2}=0.47\right)$.

Further, we might also expect a correlation of $Z^{\prime}$ anomalies, and also of stratospheric warming, with the changes in the zonal winds. We use winds at $100 \mathrm{hPa}$ because at this level the zonal mean response is largest (Fig. 1). Figures 9c and 9e show that the correlations of the subtropical wind changes at $100 \mathrm{hPa}$ averaged over $20^{\circ}-40^{\circ} \mathrm{N}$ with both $Z^{\prime}$ and polar stratospheric warming are significant $(p=0.05)$ in AMIP4K with correlation 
a) T*@20hPa vs Z’@500hPa, amip4K

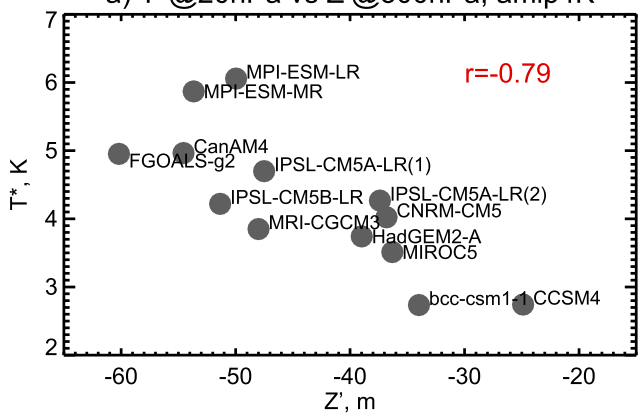

c) Z'@500hPa vs U@100hPa, amip4K

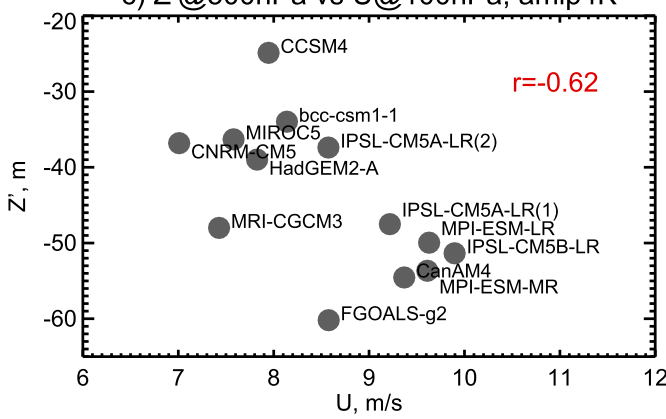

e) T*@20hPa vs U@100hPa, amip4K

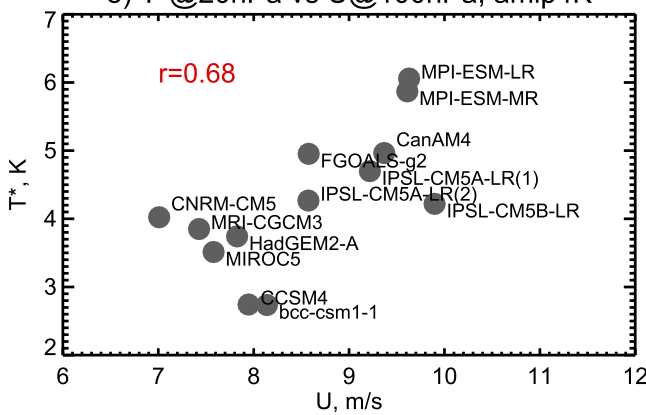

b) T*@20hPa vs Z’@500hPa, amipFuture

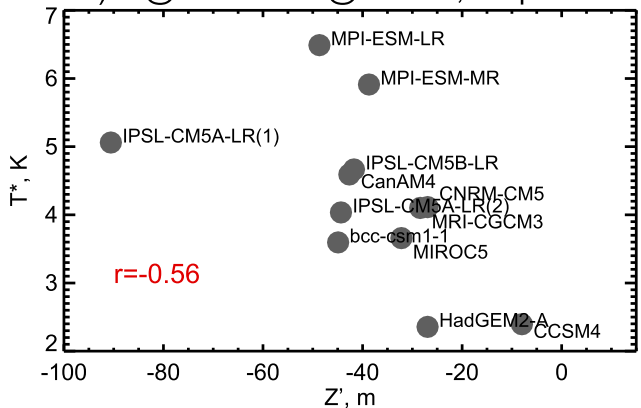

d) Z'@500hPa vs U@100hPa, amipFuture

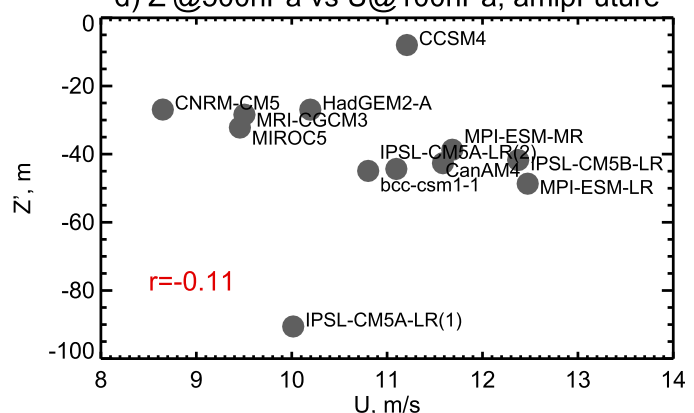

f) T*@20hPa vs U@100hPa, amipFuture

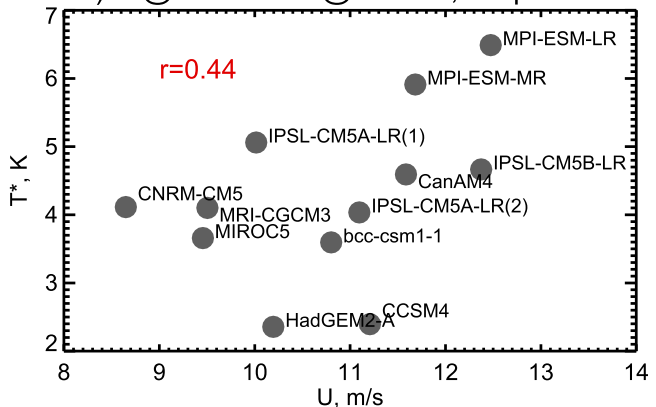

FIG. 9. Scatterplots between (a),(b) $T^{*}$ change at $20 \mathrm{hPa}$ and $Z^{\prime}$ anomaly at $500 \mathrm{hPa}$ in the North Pacific; (c),(d) zonal wind $U$ change at $100 \mathrm{hPa}$ averaged over $20^{\circ}-40^{\circ} \mathrm{N}$ and $Z^{\prime}$ anomaly at $500 \mathrm{hPa}$ in the North Pacific; and (e),(f) $T^{*}$ at $20 \mathrm{hPa}$ and $U$ wind change at $100 \mathrm{hPa}$ for (left) AMIP4K and (right) AMIPFuture.

coefficients exceeding 0.6 in absolute values. In AMIPFuture, the correlation with the $Z^{\prime}$ anomalies is again weakened by the single IPSL-CM5A-LR outlier (Fig. 9d). Excluding this simulation would improve the correlation coefficient between subtropical winds and $Z^{\prime}$ in the North Pacific from -0.11 to -0.47 . However, the correlations are in general weaker for AMIPFuture than for AMIP4K (Fig. 9f). Other factors may have contributed to the intermodel spread, although the internal variability is likely to play an important role.

It is also of interest to analyze if the spread in model responses can be linked to model biases in present climate. Scaife et al. (2012) and Karpechko and Manzini (2012) showed different responses of the polar stratosphere between models that fully resolve and do not resolve the stratosphere. Models that do not fully resolve the stratosphere typically underestimate stratospheric variability in the present climate (Dall'Amico et al. 2010; Charlton-Perez et al. 2013). Thus we address the following question: How does the spread in the magnitude of the polar warming compare to the spread in the magnitude of the present-day (unforced) variability across the models?

As a measure of the unforced variability we use the magnitude of the first empirical orthogonal function (EOF) of the $T^{*}$ variability in the present climate. The calculations have been done using deseasonalized monthly $T^{*}$ data using only December, January, and February. The first EOF of $T^{*}$ at $20 \mathrm{hPa}$ for the models and ERA-I, calculated as projections of $T^{*}$ time series on the normalized first principal component time series, are shown in Fig. 2d. Comparing Fig. 2d with Figs. 2a and 2b 
a) $\mathrm{T}^{\star} @ 20 \mathrm{hPa}$ vs EOF, amip4K

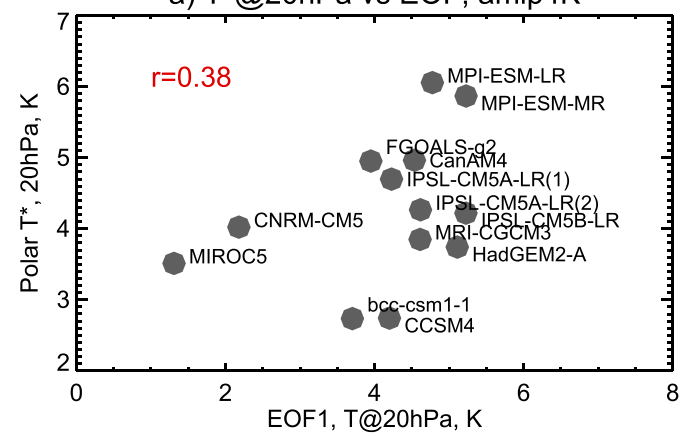

b) $T^{\star} @ 20 h P a$ vs EOF, amipFuture

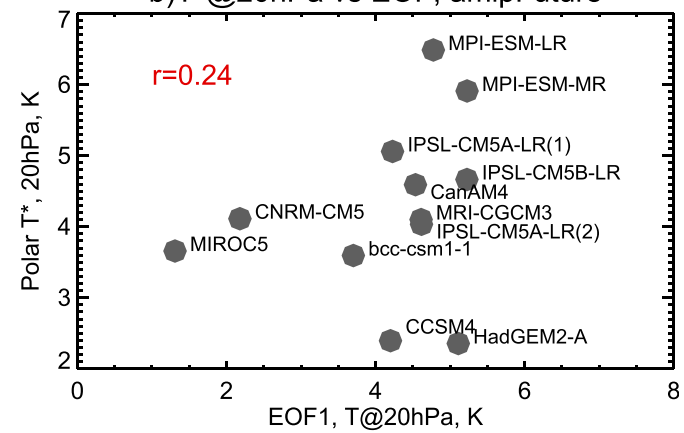

FIG. 10. Scatterplots between $T^{*}$ change at $20 \mathrm{hPa}$ and first EOF magnitude for (a) AMIP4K and (b) AMIPFuture.

shows that the stratospheric response to global warming strongly projects on the first EOF. In all models, as well as in ERA-I, the mode is a dipole with the node located between $35^{\circ}$ and $50^{\circ} \mathrm{N}$ depending on model. The mode explains $70 \%-90 \%$ of the monthly variability in the AMIP experiment depending on model, which is somewhat larger than in ERA-I (64\%). The magnitude of the mode in the polar stratosphere in BCC_CSM1.1, FGOALS-g2, CCSM4, and MIROC5 is considerably smaller than that in ERA-I, suggesting a lack of unforced variability. These models have the upper model level below the stratopause; that is, they do not fully resolve the stratosphere, thus an underestimation of the variability in these models is expected (Charlton-Perez et al. 2013). Note that the amplitude of the first EOF averaged over $60^{\circ}-90^{\circ} \mathrm{N}$ is tightly correlated with the standard deviation of the monthly $T^{*}$ values averaged over the same latitudes. Thus, if the stratospheric response to SST warming is related to unforced variability, which is underestimated in some models, then one may expect a correlation between the magnitude of the response and the magnitude of the first EOF.

The correlation between the magnitude of the first EOF in the Arctic stratosphere and the stratospheric warming response in AMIP4K and AMIPFuture is shown in Fig. 10. Although the tendency for models with small unforced variability to simulate a smaller warming is evident, the correlation coefficients are less than 0.4 in both experiments.

Since the unforced stratospheric variability and future change in North Pacific $Z^{\prime}$ are two independent factors contributing to the intermodel spread in future stratospheric warming, one is tempted to apply a multiple linear regression (i.e., Karpechko et al. 2013) to see how much of the intermodel spread can be explained by these two factors. We find, however, that a multiple regression adds negligible improvements to the linear correlation with the $Z^{\prime}$ anomalies. On the other hand the number of models may be too small to use more than one explanatory variable (i.e., to rely on a multiple regression approach).

Finally, we would like to emphasize that the strongly nonlinear nature of extratropical stratospheretroposphere circulation and its large variability imply that the dynamical response to global warming will likely contain a considerable unpredictable component. Nevertheless, the tight link between $Z^{\prime}$ anomalies in the North Pacific and future stratospheric warming provides strong evidence that the changes in the tropospheric stationary waves are responsible for driving future stratospheric changes.

\section{Discussion and conclusions}

Climate models often simulate dynamical warming of the Arctic winter stratosphere as a response to global warming in association with a strengthening of the deep branch of the BD circulation. However, until now, no satisfactory mechanism has been suggested on the strengthening of the deep branch of the BD circulation, casting doubts as to the realism of the response. Here we show that a mechanism for strengthening of the BD circulation in global warming experiments with prescribed SSTs involves an increased wave activity flux into the stratosphere due to planetary wavenumber 1 . We propose that this increased wave activity flux is ultimately related to the strengthening of the tropospheric zonal winds with global warming, which is a robust change. The strengthening of the tropospheric zonal wind can in turn lead to enhanced stationary wavenumber 1 activity both by enhanced generation by orography and by the wave-lengthening effect. Note that although we demonstrate a relation between winds strengthening and increases in stationary wave amplitude, we cannot rule out alternative sources of stationary wave activity, in particular those related to tropical and extratropical diabatic heating anomalies and 
transient eddy fluxes, whose relative role remains to be understood. The key region for the upward influence of the tropospheric wave response is the North Pacific, which is consistent with the location of tropospheric precursors of the stratospheric polar vortex variability at the intraseasonal time scale as is shown here and, for instance, in Garfinkel et al. (2010). In particular, we show that the eastward shift and deepening of the climatological low over the North Pacific is consistent with increased wavenumber 1 heat flux into the stratosphere as well as the Arctic stratospheric warming.

All models reproduce strengthening of the upper troposphere and lower stratosphere (UTLS) winds, deepening of the geopotential heights over the North Pacific, and warming of the Arctic stratosphere. As a general rule, models with larger changes in the winds also simulate larger changes in the tropospheric waves and consequently larger stratospheric warming, which supports the mechanism outlined above. Internal variability is likely to play a large role in the extratropical circulation response; however, the lack of ensemble simulations limits our ability to quantify its role. The similarity of the responses between AMIP4K and AMIPFuture suggests that the large-scale atmospheric circulation response is not too sensitive to details of future SST warming. It is worth noting that the SST changes observed during 1979-2009 induced weakening, rather than strengthening, of stratospheric wavenumber 1 , as seen in observations and climate model simulations (Garfinkel et al. 2015). However, these SST changes include both warming and cooling regions and are likely strongly influenced by natural decadal variability, which is expected to be overwhelmed by climate change warming signal at some point in the future. The responses in AMIP4K and AMIPFuture are contrasted with that in AMIP4 $\times \mathrm{CO}_{2}$, which lacks tropical amplification of the warming and strengthening of the uppertropospheric winds in the subtropics and midlatitudes. The lack of Arctic stratospheric warming in this experiment is consistent with the lack of tropospheric wave response.

The use of simplified global warming experiments adopted in this paper removes uncertainty associated with poorly constrained climate sensitivity and details of SST changes but also introduces some caveats. The lack of the Arctic amplification could be an important one, given that concomitant changes related to it can affect the forcing of the stationary planetary waves. Although weakening of the polar vortex and a strengthening of the BD circulation has been found in CMIP5 model simulations following the RCP8.5 scenario (Manzini et al. 2014), the models showed a larger spread in the stratospheric response in comparison to the present study, partly because more models were available for analysis. It would be interesting to understand how full atmosphere-ocean coupling impacts on the tropospheric wave changes over the North Pacific, which is found to be the key region influencing future stratospheric changes. Such a work is planned for the future.

Finally we note that, although the uncertainty in future Arctic stratospheric changes is largely due to chaotic atmospheric circulation variability in the extratropics, the main result of our work is that changes in tropospheric stationary wave patterns are consistent with weakening of the Arctic vortex in the future, providing a physical explanation for the strengthening of the deep branch of the BD circulation and dynamical warming of the Arctic stratosphere, which are often simulated by climate models.

Acknowledgments. We acknowledge the World Climate Research Programme (WCRP) Working Group on Coupled Modelling (WGCM), which is responsible for CMIP, and we thank the climate modeling groups for producing and making available their model output. We thank ECMWF for providing access to ERA-Interim. We thank three anonymous reviewers for helpful comments on earlier version of the manuscript and Isla Simpson, Evgeny Kadantsev, and Nicholas Tyrrell for useful discussions. The work of AK is supported by the Academy of Finland (Grants 286298 and 294120).

\section{REFERENCES}

Birner, T., and H. Bönisch, 2011: Residual circulation trajectories and transit times into the extratropical lowermost stratosphere. Atmos. Chem. Phys., 11, 817-827, doi:10.5194/acp-11-817-2011.

Butchart, N., 2014: The Brewer-Dobson circulation. Rev. Geophys., 52, 157-184, doi:10.1002/2013RG000448.

_- J. Austin, J. R. Knight, A. A. Scaife, and M. L. Gallani, 2000: The response of the stratospheric climate to projected changes in the concentrations of well-mixed greenhouse gases from 1992 to 2051. J. Climate, 13, 2142-2159, doi:10.1175/ 1520-0442(2000)013<2142:TROTSC $>2.0 . C O ; 2$.

Calvo, N., and R. R. Garcia, 2009: Wave forcing of the tropical upwelling in the lower stratosphere under increasing concentrations of greenhouse gases. J. Atmos. Sci., 66, 3184-3196, doi:10.1175/2009JAS3085.1.

Charlton-Perez, A. J., and Coauthors, 2013: On the lack of stratospheric dynamical variability in low-top versions of the CMIP5 models. J. Geophys. Res. Atmos., 118, 2494-2505, doi:10.1002/jgrd.50125.

Dall'Amico, M., P. A. Stott, A. A. Scaife, L. J. Gray, K. H. Rosenlof, and A. Yu. Karpechko, 2010: Impact of stratospheric variability on tropospheric climate change. Climate Dyn., 34, 399-417, doi:10.1007/s00382-009-0580-1.

Deser, C., R. Tomas, M. Alexander, and D. Lawrence, 2010: The seasonal atmospheric response to projected Arctic sea ice loss in the late twenty-first century. J. Climate, 23, 333-351, doi:10.1175/2009JCLI3053.1. 
Eichelberger, S. J., and D. L. Hartmann, 2005: Changes in the strength of the Brewer-Dobson circulation in a simple AGCM. Geophys. Res. Lett., 32, L15807, doi:10.1029/2005GL022924.

Engel, A., and Coauthors, 2009: Age of stratospheric air unchanged within uncertainties over the past 30 years. Nat. Geosci., 2, 28-31, doi:10.1038/ngeo388.

Eyring, V., and Coauthors, 2007: Multimodel projections of stratospheric ozone in the 21st century. J. Geophys. Res., 112, D16303, doi:10.1029/2006JD008332.

Fioletov, V. E., J. B. Kerr, and D. I. Wardle, 1997: The relationship between total ozone and spectral UV irradiance from Brewer observations and its use for derivation of total ozone from UV measurements. Geophys. Res. Lett., 24, 2997-3000, doi:10.1029/ 97GL53153.

Forster, P. M. F., and K. P. Shine, 2002: Assessing the climate impact of trends in stratospheric water vapor. Geophys. Res. Lett., 29, 1086, doi:10.1029/2001GL013909.

Fu, Q., P. Lin, S. Solomon, and D. L. Hartmann, 2015: Observational evidence of strengthening of the Brewer-Dobson circulation since 1980. J. Geophys. Res. Atmos., 120, 10 214-10228, doi:10.1002/2015JD023657.

Garcia, R. R., and W. J. Randel, 2008: Acceleration of the BrewerDobson circulation due to increases in greenhouse gases. J. Atmos. Sci., 65, 2731-2739, doi:10.1175/2008JAS2712.1.

Garfinkel, C. I., D. L. Hartmann, and F. Sassi, 2010: Tropospheric precursors of anomalous Northern Hemisphere stratospheric polar vortices. J. Climate, 23, 3282-3299, doi:10.1175/2010JCLI3010.1.

—, M. M. Hurwitz, and L. D. Oman, 2015: Effect of recent sea surface temperature trends on the Arctic stratospheric vortex. J. Geophys. Res. Atmos., 120, 5404-5416, doi:10.1002/2015JD023284.

Grise, K. M., and L. M. Polvani, 2014: The response of midlatitude jets to increased $\mathrm{CO}_{2}$ : Distinguishing the roles of sea surface temperature and direct radiative forcing. Geophys. Res. Lett., 41, 6863-6871, doi:10.1002/2014GL061638.

Hegglin, M. I., and T. G. Shepherd, 2009: Large climate-induced changes in ultraviolet index and stratosphere-to-troposphere ozone flux. Nat. Geosci., 2, 687-691, doi:10.1038/ngeo604.

Held, I. M., 1983: Stationary and quasi-stationary eddies in the extratropical atmosphere: Theory. Large Scale Dynamical Processes in the Atmosphere, R. P. Pearce and B. J. Hoskins, Eds., Academic Press, 127-168.

—- M. Ting, and H. Wang, 2002: Northern winter stationary waves: Theory and modeling. J. Climate, 15, 2125-2144, doi:10.1175/1520-0442(2002)015<2125:NWSWTA >2.0.CO;2.

Holton, J. R., P. H. Haynes, M. E. McIntyre, A. R. Douglass, R. B. Rood, and L. Pfister, 1995: Stratosphere-troposphere exchange. Rev. Geophys., 33, 403-439, doi:10.1029/95RG02097.

Karpechko, A. Yu., and E. Manzini, 2012: Stratospheric influence on tropospheric climate change in the Northern Hemisphere. J. Geophys. Res., 117, D05133, doi:10.1029/2011JD017036.

- D. Maraun, and V. Eyring, 2013: Improving Antarctic total ozone projections by a process-oriented multiple diagnostic ensemble regression. J. Atmos. Sci. 70, 3959-3976, doi:10.1175/JAS-D-13-071.1.

Kidston, J., A. A. Scaife, S. C. Hardiman, D. M. Mitchell, N. Butchart, M. P. Baldwin, and L. J. Gray, 2015: Stratospheric influence on tropospheric jet streams, storm tracks and surface weather. Nat. Geosci., 8, 433-440, doi:10.1038/ngeo2424.

Manzini, E., and Coauthors, 2014: Northern winter climate change: Assessment of uncertainty in CMIP5 projections related to stratosphere-troposphere coupling. J. Geophys. Res. Atmos., 119, 7979-7998, doi:10.1002/2013JD021403.

McLandress, C., and T. G. Shepherd, 2009: Simulated anthropogenic changes in the Brewer-Dobson circulation, including its extension to high latitudes. J. Climate, 22, 1516-1540, doi:10.1175/2008JCLI2679.1.

Neu, J. L., T. Flury, G. L. Manney, M. L. Santee, N. J. Livesey, and J. Worden, 2014: Tropospheric ozone variations governed by changes in stratospheric circulation. Nat. Geosci., 7, 340-344, doi:10.1038/ngeo2138.

Newman, P. A., E. R. Nash, and J. E. Rosenfield, 2001: What controls the temperature of the Arctic stratosphere during the spring? J. Geophys. Res., 106, 19 999-20 010, doi:10.1029/2000JD000061.

Oberländer, S., U. Langematz, and S. Meul, 2013: Unraveling impact factors for future changes in the Brewer-Dobson circulation J. Geophys. Res. Atmos., 118, 10296-10312, doi:10.1002/jgrd.50775. , and Coauthors, 2016: Is the Brewer-Dobson circulation increasing or moving upward? Geophys. Res. Lett., 43, 17721779, doi:10.1002/2015GL067545.

Olsen, M. A., M. R. Schoeberl, and J. E. Nielsen, 2007: Response of stratospheric circulation and stratosphere-troposphere exchange to changing sea surface temperatures. J. Geophys. Res., 112, D16104, doi:10.1029/2006JD008012.

Orsolini, Y. J., A. Yu. Karpechko, and G. Nikulin, 2009: Variability of the Northern Hemisphere polar stratospheric cloud potential: The role of North Pacific disturbances. Quart. J. Roy. Meteor. Soc., 135, 1020-1029, doi:10.1002/qj.409.

Plumb, R. A., 2002: Stratospheric transport. J. Meteor. Soc. Japan, 80, 793-801, doi:10.2151/jmsj.80.793.

Scaife, A. A., and Coauthors, 2012: Climate change projections and stratosphere-troposphere interaction. Climate Dyn., 38, 20892097, doi:10.1007/s00382-011-1080-7.

Shepherd, T. G., 2014: Atmospheric circulation as a source of uncertainty in climate change projections. Nat. Geosci., 7, 703708, doi:10.1038/ngeo2253.

_ , and C. McLandress, 2011: A robust mechanism for strengthening of the Brewer-Dobson circulation in response to climate change: Critical-layer control of subtropical wave breaking. J. Atmos. Sci., 68, 784-797, doi:10.1175/2010JAS3608.1.

Sigmond, M., and J. F. Scinocca, 2010: The influence of the basic state on the Northern Hemisphere circulation response to climate change. J. Climate, 23, 1434-1446, doi:10.1175/2009JCLI3167.1.

— , P. C. Siegmund, E. Manzini, and H. Kelder, 2004: A simulation of the separate climate effects of middle atmospheric and tropospheric $\mathrm{CO}_{2}$ doubling. J. Climate, 17, 2352-2367, doi:10.1175/1520-0442(2004)017<2352:ASOTSC > 2.0.CO;2.

Simpson, I. R., R. Seager, M. Ting, and T. A. Shaw, 2016: Causes of change in Northern Hemisphere winter meridional winds and regional hydroclimate. Nat. Climate Change, 6, 65-70, doi:10.1038/nclimate2783.

Thompson, D. W. J., J. M. Wallace, and G. C. Hegerl, 2000: Annular modes in the extratropical circulation. Part II: Trends. J. Climate, 13, 1018-1036, doi:10.1175/1520-0442(2000)013<1018: AMITEC $>2.0 . \mathrm{CO} ; 2$.

Ueyama, R., and J. M. Wallace, 2010: To what extent does high-latitude wave forcing drive tropical upwelling in the Brewer-Dobson circulation? J. Atmos. Sci., 67, 1232-1246, doi:10.1175/2009JAS3216.1.

Woollings, T., 2008: Vertical structure of anthropogenic zonalmean atmospheric circulation change. Geophys. Res. Lett., 35 , L19702, doi:10.1029/2008GL034883.

_ J. M. Gregory, J. G. Pinto, M. Reyers, and D. J. Brayshaw, 2012: Response of the North Atlantic storm track to climate change shaped by ocean-atmosphere coupling. Nat. Geosci., $\mathbf{5}$, 313-317, doi:10.1038/ngeo1438.

Yulaeva, E., J. R. Holton, and J. M. Wallace, 1994: On the cause of the annual cycle in tropical lower-stratospheric temperatures. J. Atmos. Sci., 51, 169-174, doi:10.1175/ 1520-0469(1994)051<0169:OTCOTA >2.0.CO;2. 